\title{
STRATEGIES FOR SETTING THE PIP LIMIT DURING VOLUME GUARANTEE VENTILATION
}

\author{
K.I. Wheeler ${ }^{1,2,3}$, C. Wong ${ }^{1}$, C.J. Morley ${ }^{1}$, P.G. Davis ${ }^{1,4,5}$
}

${ }^{I}$ Newborn Research, Royal Women's Hospital, ${ }^{2}$ NMHRC CCRE in Newborn Medicine. RWH, ${ }^{3}$ Ritchie Center for Medical Research, Monash University, ${ }^{4}$ University of Melbourne, ${ }^{5}$ Murdoch Childrens Research Institute, Melbourne, VIC, Australia

In volume guarantee (VG) mode, the Dräger Babylog 8000plus ventilator targets a set expired tidal volume $\left(\mathrm{V}_{\mathrm{T}}\right)$ by adjusting the PIP between the positive end expiratory pressure (PEEP) and the maximum PIP limit (Pmax). If Pmax is too low, $\mathrm{V}_{\mathrm{T}}$ may not be delivered and the ventilator alarms. If too high, infants may be at risk of unnecessarily high inflation pressures and $\mathrm{V}_{\mathrm{T}}$. Three strategies for selecting Pmax were compared.

Infants were eligible if stable on assist control (AC) VG for the previous 24 hours. Three strategies for setting Pmax were compared during 20 minute epochs in random order: $\mathrm{F}=$ Fixed unit setting $\left(30 \mathrm{~cm}_{2} \mathrm{O}\right)$; $\mathrm{C}=$ Pmax set $5 \mathrm{~cm} \mathrm{H}_{2} \mathrm{O}$ above the typical peak pressure reported by the clinical staff; $\mathrm{T}=\mathrm{Pmax}$ set $5 \mathrm{~cm} \mathrm{H}_{2} \mathrm{O}$ above the average PIP during triggered ventilation. Ventilator data and measures of cardiorespiratory stability were recorded. Analysis was repeated excluding spontaneous large breaths.

15 infants were studied. Median (IQR) corrected gestation, weight and $\mathrm{FiO}_{2}$ were $27(25,30)$ weeks, 0.78 $(0.73,1.5) \mathrm{kg}$ and $0.23(0.21,0.31)$ respectively. Mean $(\mathrm{SD})$ results shown below:

\begin{tabular}{|c|c|c|c|c|c|}
\hline & F (Fixed) & C (Clinical+5) & T (Triggered+5) & $\begin{array}{c}\text { Regression } \\
\text { (F v C) p value }\end{array}$ & $\begin{array}{c}\text { Regression } \\
\text { (F v T) p value }\end{array}$ \\
\hline Pmax (cm H2O) & $30(0)$ & $23(5)$ & $21(5)$ & & 0.52 \\
\hline PIP (cm H2O) & $19(5)$ & $17(5)$ & $18(6)$ & 0.12 & 0.14 \\
\hline $\begin{array}{c}\text { VT }>\mathbf{2 0 0 \%} \\
\text { target (all 54197 } \\
\text { inflations) }\end{array}$ & $4.0(3.4)$ & $2.6(3.1)$ & $3.0(2.4)$ & 0.03 & 0.02 \\
\hline $\begin{array}{c}\text { VT>200\% } \\
\text { target (47214 } \\
\text { supported } \\
\text { inflations) }\end{array}$ & $4.0(3.3)$ & $2.4(2.7)$ & $2.4(1.9)$ & 0.05 & \\
\hline [Results] & & & \\
\hline
\end{tabular}

Cardiorespiratory parameters were stable at all settings.

Strategies which set Pmax $5 \mathrm{~cm} \mathrm{H}_{2} \mathrm{O}$ above the triggered PIP reduced the proportion of inflations with $\mathrm{V}_{\mathrm{T}}>200 \%$ target. 